

\title{
Mobilité de hall dans le bromure de césium
}

\author{
A. Bruaux, J. Claus, G. Moens
}

\section{To cite this version:}

A. Bruaux, J. Claus, G. Moens. Mobilité de hall dans le bromure de césium. Revue de Physique Appliquée, 1968, 3 (1), pp.67-72. 10.1051/rphysap:019680030106700 . jpa-00242825

\section{HAL Id: jpa-00242825 https://hal.science/jpa-00242825}

Submitted on 1 Jan 1968

HAL is a multi-disciplinary open access archive for the deposit and dissemination of scientific research documents, whether they are published or not. The documents may come from teaching and research institutions in France or abroad, or from public or private research centers.
L'archive ouverte pluridisciplinaire HAL, est destinée au dépôt et à la diffusion de documents scientifiques de niveau recherche, publiés ou non, émanant des établissements d'enseignement et de recherche français ou étrangers, des laboratoires publics ou privés. 


\title{
MOBILITÉ DE HALL DANS LE BROMURE DE GÉSIUM (1)
}

\author{
Par A. BRUAUX, J. GLAUS, G. MOENS, \\ Laboratorium voor Kristallografie en Studie van de Vaste Stof, Rijksuniversiteit, Gent (Belgie). \\ (Reçu le 5 juin 1967.)
}

\begin{abstract}
Résumé. - La mobilité de Hall dans le bromure de césium a été mesurée entre les températures de $10 \mathrm{o}$ et $50 \mathrm{oK}$ par la méthode proposée par Redfield et modifiée par Brown. La mobilité vaut environ $8000 \mathrm{~cm}^{2} / V$.s aux basses températures. Une théorie du signal observé dans ces expériences est donnée et confirme la formulation de Brown. Des recherches ultérieures seraient nécessaires pour interpréter complètement nos résultats.
\end{abstract}

Abstract. - The Hall mobility in caesium bromide has been measured between 10 and $50{ }^{\circ} \mathrm{K}$ by the Redfield method as modified by Brown. The mobility is about $8000 \mathrm{~cm}^{2} / \mathrm{V} . \mathrm{s}$ at low temperature. A theory of the signal observed in these experiments is given and confirms Brown's formulation. Further research would be necessary to interpret our results more completely.

I. Introduction. - Nous avons mesuré la mobilité de Hall dans le CsBr par la méthode de Brown [1]. Gette méthode est une modification de celle proposée par Redfield [2] qui a étudié la mobilité de Hall dans le diamant. La figure 1 est un schéma de principe du montage.

La méthode se distingue par le fait qu'elle est impulsionnelle et que le cristal est complètement isolé de façon à éviter l'injection de porteurs aux contacts. La méthode permet ainsi d'éliminer plus facilement l'influence de charges spatiales. Elle convient bien à l'étude de la mobilité des photoélectrons dans les matériaux de haute résistivité.

Lorsque la tension, appliquée par impulsion, passe par son maximum, une étincelle $\mathrm{F}$ de durée environ

(1) Ce travail a été réalisé sous les auspices de I'I.R.S.I.A mille fois plus courte que cette impulsion éclate entre des électrodes dans l'air. Pendant la durée de cette étincelle, on peut supposer la tension constante et par conséquent qu'il en est de même pour le champ électrique en tout point de l'espace situé entre la couche résistive $\mathrm{AB}$ et l'électrode métallique ( $f g .1$ ) reliée à l'entrée du préamplificateur. Cet espace est occupé par le cristal, par une feuille en mylar qui isole le cristal de l'électrode métallique et par la lame de verre qui supporte l'électrode résistive. Les photons, provenant de l'étincelle, ionisent les centres $F$ et les électrons ainsi libérés passent dans la bande de conduction. Sous l'action du champ électrique, ces électrons sont mis en mouvement. Les variations de charges sont alors induites sur les électrodes et un signal est donc engendré.

Si l'on suppose que les électrons sont libérés en densité uniforme dans le cristal et que leur mouvement est

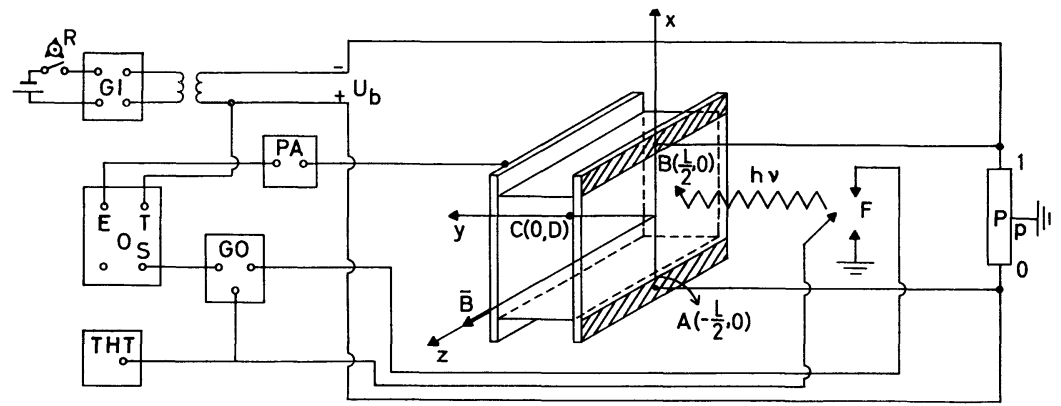

FIG. 1. - Schéma de principe. - GI : générateur d'impulsions; GO : générateur d'ondes à front raide ; $\mathrm{PA}$ : préamplificateur; $\mathrm{O}$ : oscilloscope ; $\mathrm{E}$ : entrée de l'oscilloscope ; $\mathrm{T}$ : trigger ; $\mathrm{S}$ : synchronisation ; $\mathrm{P}$ : potentiomètre; $\mathrm{F}$ : éclair lumineux, et $\mathrm{R}$ : interrupteur rotatif. 
seulement dû à l'action du champ électrique appliqué extérieurement, on montre qu'il existe une position $p_{1}$ $\mathrm{du}$ curseur du potentiomètre $\mathrm{P}$ pour laquelle le signal est nul. (En modifiant la position du curseur, on fait varier d'une même quantité la valeur du potentiel par rapport à la masse, de chaque point de la couche résistive. Cette quantité est proportionnelle au déplacement du curseur.)

Si, pour cette position du curseur, on refait l'expérience cette fois en appliquant un champ magnétique selon la direction indiquée sur la figure 1, les électrons subissent en plus l'action d'une force de Lorentz. De ce fait, un signal réapparaît.

On montre, dans les conditions citées précédemment, qu'il existe aussi une position $p_{2}$ du curseur pour laquelle le signal est nul en présence de champ magnétique. La mesure de $p_{1}-p_{2}$ entre les expériences avec et sans champ magnétique est une mesure, à une constante près, de la mobilité de Hall.

Pour le lecteur désirant avoir plus de détails, nous renvoyons à [1].

Brown et coll. [3], [4], [5] et [6] ont étudié les chlorure et bromure d'argent, Kobayashi et coll. se sont intéressés au CdS [7]. Nous avons également adopté cette méthode pour l'étude de la mobilité dans le $\mathrm{CsBr}$.

II. Théorie du signal. - La méthode de Brown est basée sur l'annulation du signal de photoconduction avec et sans champ magnétique alternativement. Comme nous n'avons réussi qu'à annuler la première partie de ce signal, nous avons été conduits à entreprendre son étude théorique. Ce signal est engendré par un courant de diffusion et de conduction dans le cristal.

Les hypothèses de départ sont les suivantes :

$1^{\circ}$ Il n'existe qu'un seul type de porteurs mobiles (ici, électrons);

$2^{\circ}$ Le seul champ électrique agissant sur les porteurs est celui appliqué de l'extérieur (pas de champ dû aux charges spatiales ou superficielles) et est supposé constant pendant le temps relativement court que dure l'étincelle;

$3^{o}$ Nous négligeons la différence de permittivité entre le cristal et le vide, ainsi que les effets de bord dans la recherche de l'expression analytique du champ électrique;

$4^{\text {o }}$ Le champ magnétique est homogène;

$5^{\circ}$ L'effet de diffusion des porteurs est négligé;

$6^{\circ}$ Un porteur atteignant une surface y est directement piégé;

$7^{0}$ L'intensité lumineuse décroît exponentiellement dans le cristal (on néglige les réflexions possibles). La lumière est monochromatique;

$8^{\circ}$ On suppose $\mu=\mu_{d}=\mu_{H}$ pour la simplicité du calcul (mobilités de transport et de Hall).
De plus, ces mobilités sont supposées constantes.

Le signal capté par le préamplificateur dépend de la variation de charge sur l'électrode métallique sous l'influence du mouvement des porteurs ainsi que l'indique la formule suivante [2] :

$$
I(t)=\frac{\delta Q^{\prime}}{\delta t}=q \iiint_{\text {cristal }} n \frac{v_{y}}{D} \mathrm{~d} x \mathrm{~d} y \mathrm{~d} z
$$

où $n$ désigne la densité des porteurs, $v_{y}$ leur vitesse suivant l'axe. La variation $\delta Q^{\prime}$ est accompagnée d'une variation $\delta Q^{\prime \prime}$ de la charge sur l'électrode métallique sous l'influence de la variation de potentiel de cette électrode.

La quantité $I(t)$ ne constitue donc nullement le signal observable sur l'oscilloscope. En effet, ce potentiel varie, l'admittance du circuit relié à la capacité $C_{\mathrm{e}}$ formée par cette électrode et l'électrode transparente n'étant pas nulle. Cette admittance, en parallèle sur $C_{\mathrm{c}}$, est constituée par une capacité parasite shuntée par l'impédance d'entrée du préamplificateur.

On peut alors calculer analytiquement la tension d'entrée du préamplificateur par l'intermédiaire de l'intégrale de convolution :

$$
U(t)=\int_{0}^{t} I(t) u\left(t-t^{\prime}\right) \mathrm{d} t^{\prime}
$$

où $u(t)$ est la réponse du circuit sous l'influence d'une impulsion de courant (fonction de Dirac). Ensuite, il faut tenir compte de la fonction de transfert de la chaîne d'amplification. Une nouvelle intégrale de convolution donne la réponse $V(t)$ à l'oscilloscope pour la tension d'entrée $U(t)$.


électrique sont données par :

$$
\begin{aligned}
& E_{x}=-\frac{U_{b}}{L D}(y-D) \\
& E_{y}=-\frac{U_{b}}{L D} x+\frac{U_{b}}{D}(p-1 / 2)
\end{aligned}
$$

où $p$ est un nombre déterminant la position du potentiomètre (fig. 1).

L'évaluation de l'intégrale (1) se fait par le truchement d'une troisième intégrale de convolution. On suppose tout d'abord que l'intensité lumineuse se représente par une fonction de Dirac en $t$.

Dès lors, utilisant les lois classiques du mouvement d'un porteur (plongé ou non dans un champ magnétique) et l'équation de continuité pour la densité $n$ de porteurs en l'absence de génération, on arrive à la conclusion que la densité en électrons décroît exponentiellement au cours du temps, à condition de suivre les électrons dans leur mouvement :

$$
n(x, y, t)=n\left(x_{0}, y_{0}, 0\right) \mathrm{e}^{-t / \tau}
$$

où $\tau$ est la durée de vie moyenne des porteurs dans le cristal, les relations entre $(x, y)$ et $\left(x_{0}, y_{0}\right)$ étant données par les lois du mouvement (la coordonnée $z$ n'intervient pas puisqu'il n'est supposé aucun mouvement dans cette direction). 
Dans le cas de la figure 1, la densité initiale en porteurs est décrite par :

$$
n\left(x_{0}, y_{0}, 0\right)=N_{0} \mathrm{e}^{-r y_{0}}
$$

où $r$ est le coefficient d'absorption de la lumière dans le cristal. Il est clair que la solution (4) n'est pas valable dans tout le cristal. En effet, le nuage de porteurs libres est balayé par l'action combinée des champs électrique et magnétique et à chaque instant on peut définir une surface qui sépare la région d'où les électrons libres ont disparu, en raison de leur mouvement, de la région où il en subsiste. Dans la première région, la densité $n$ est nulle; pour l'autre, l'expression (4) est valable. $v_{y}$ est obtenue simplement à partir des lois du mouvement.

Remplaçant dans (1) $n$ et $v_{y}$ par leurs expressions trouvées, il nous est possible de calculer l'intégrale qui est une réponse $I_{1}(t)$ pour une impulsion lumineuse brève. La réponse $I(t)$ est alors obtenue à partir du produit de convolution de la fonction $I_{1}(t)$ et de la fonction de transfert représentée par l'expression analytique de la variation dans le temps de l'intensité de l'éclair.

Nous n'avons pas reproduit ici les détails des calculs, ceux-ci étant par trop fastidieux et les expressions trop lourdes. La tabulation des diverses fonctions $V(t)$, correspondant à différentes valeurs des paramètres $r$, $\mu, \tau$ et $p$, en l'absence et en présence de champ magnétique, ainsi que l'ensemble des calculs numériques impliqués dans les convolutions, ont été effectués à l'aide de la calculatrice P3 du Laboratoire de Recherche de la M.B.L.E. $\left({ }^{2}\right)$.

Les figures 2 et 3 donnent les réponses $V(t)$ respec-

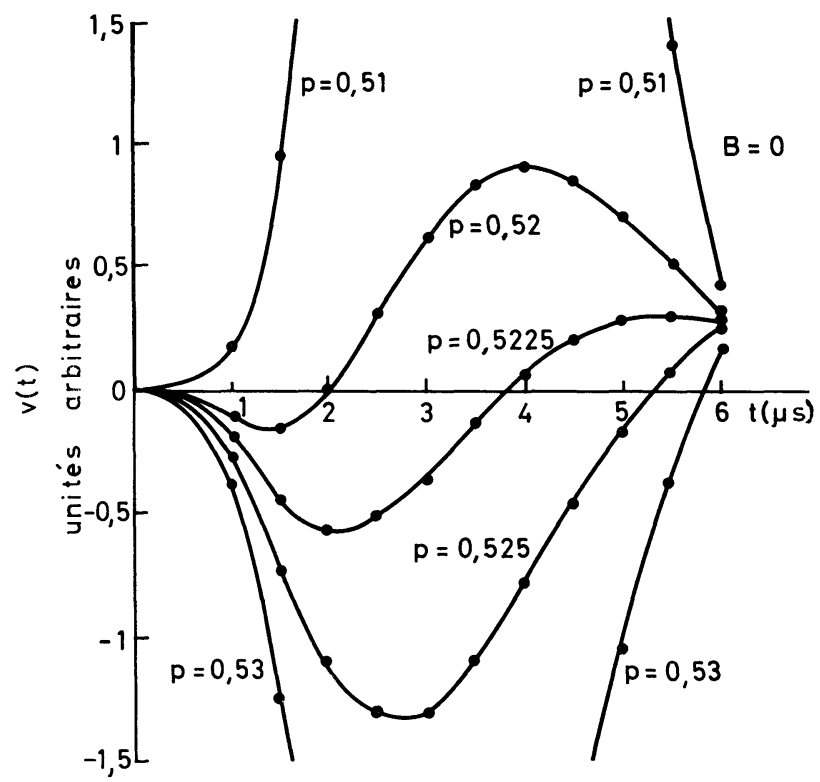

FIG. 2. $-B=0 \mathrm{~Wb} / \mathrm{m}^{2} ; U_{\mathrm{b}}=200 \mathrm{~V} ; L=1 \mathrm{~cm}$; $D=0,325 \mathrm{~cm} ; \quad r=50 \mathrm{~cm}^{-1} ; \quad \tau=10^{-7} \mathrm{~s}$; $\mu=5360 \mathrm{~cm}^{2} / \mathrm{V}$ s.

(2) Manufacture Belge de Lampes et de Matériel Electronique

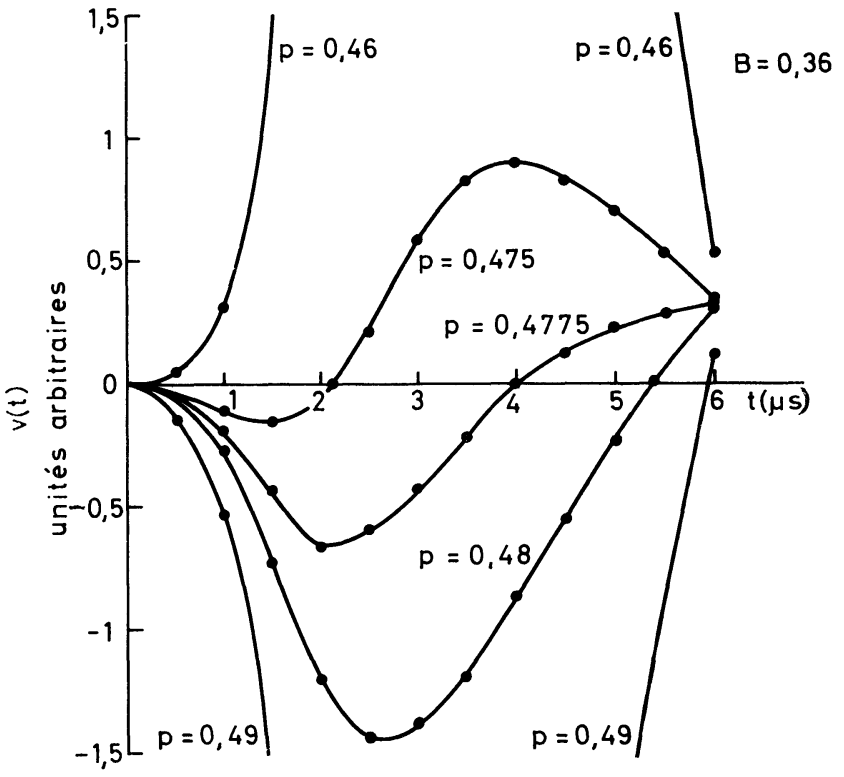

FIG. 3. - Signal de Hall : $B=0,36 \mathrm{~Wb} / \mathrm{m}^{2} ; U_{b}=200 \mathrm{~V}$; $L=1 \mathrm{~cm} ; D=0,325 \mathrm{~cm} ; r=50 \mathrm{~cm}^{-1} ; \tau=10^{-7} \mathrm{~s}$; $\mu=5360 \mathrm{~cm}^{2} /$ V.s.

tivement dans les cas où $B$ est nul, différent de zéro, ainsi que pour des valeurs de paramètres approximativement réalisables lors de mesures de mobilités dans le chlorure d'argent.

Nous avons reconnu, à travers les formes successives du signal théorique correspondant à des valeurs différentes de $p$, les caractères généraux que nous avons observés dans nos expériences, ce qui indique que le signal est surtout fonction du courant de conduction.

Sur les figures 2 et 3 , nous remarquons l'analogie de forme entre les courbes $p=0,475(B \neq 0)$, $p=0,52(B=0), p=0,4775$ et $p=0,5225$, ainsi qu'entre $p=0,48$ et $p=0,525$. A ces valeurs de $p$ correspond un signal presque nul.

Dans ce domaine de valeurs de $p$, nous pouvons donc supposer que $U(t)$ est pratiquement nul et ne varie que très peu. Négligeant l'influence de la chaîne d'amplification, à un facteur près, $U(t)$ est la tension appliquée aux bornes de la capacité $C_{0}$. On montre facilement que si cette tension est négligeable et par voie de conséquence ses variations $\left(\delta Q^{\prime \prime} \simeq 0\right.$ ), la composante de courant, dans le cristal, normale aux deux électrodes est nulle.

Nous en concluons qu'il existe un domaine de valeurs de $p$ pour lequel on peut, assez complètement, annuler le signal et que, par conséquent, dans ce domaine, la théorie de Brown est valable.

Si nous appliquons la formule :

$$
\mu_{H}\left(\frac{\mathrm{cm}^{2}}{\mathrm{~V} \cdot \mathrm{s}}\right)=\frac{10^{4}}{B\left(\mathrm{~Wb} / \mathrm{m}^{2}\right)} \frac{L}{D} \Delta p
$$


qui peut être obtenue de la formule (6) réf. [1] par une simple transformation $(3)$, nous trouvons :

$$
\mu_{H}=3840 \frac{\mathrm{cm}^{2}}{\mathrm{~V} \cdot \mathrm{s}} \text {. }
$$

Si nous comparons cette valeur de $\mu_{H}$ avec celle adoptée au préalable $\left(5360 \mathrm{~cm}^{2} / \mathrm{V} . \mathrm{s}\right)$, nous trouvons une discordance appréciable. Ceci est dû au fait que le coefficient d'absorption a une valeur élevée. Cette discordance s'amenuise au fur et à mesure que ce coefficient décroît. Expérimentalement, nous ne devons donc illuminer le cristal que dans la queue de sa bande d'absorption. De plus, de ces courbes, nous concluons qu'au voisinage de l'équilibre les différences d'ordonnée entre les diverses courbes sont sensiblement proportionnelles au $\Delta p$ correspondant.

En conclusion, nous pouvons dire qu'en bonne approximation (coefficient d'absorption faible), nous déduirons la valeur de la mobilité à partir des valeurs de $\Delta p$ pour lesquelles les signaux obtenus pour $B=0$ et $B \neq 0$ sont petits et semblables.

L'hypothèse la plus discutable est, rappelons-le, celle relative aux effets de la charge spatiale créée par les porteurs tant libres que piégés. Il importait donc d'adopter des conditions expérimentales garantissant au mieux le respect des hypothèses admises à la base de cette théorie. Ceci nous a conduits à entreprendre une série d'essais ayant pour but de mettre en évidence la charge spatiale par les effets d'un courant de diffusion.

III. Mise en évidence de la charge spatiale. Une tension $U_{p}$ étant appliquée entre l'électrode résistive et l'électrode métallique ( $f g .4$ ), une lumière rouge tombe sur un cristal de $\mathrm{CsBr}$ et libère des électrons.

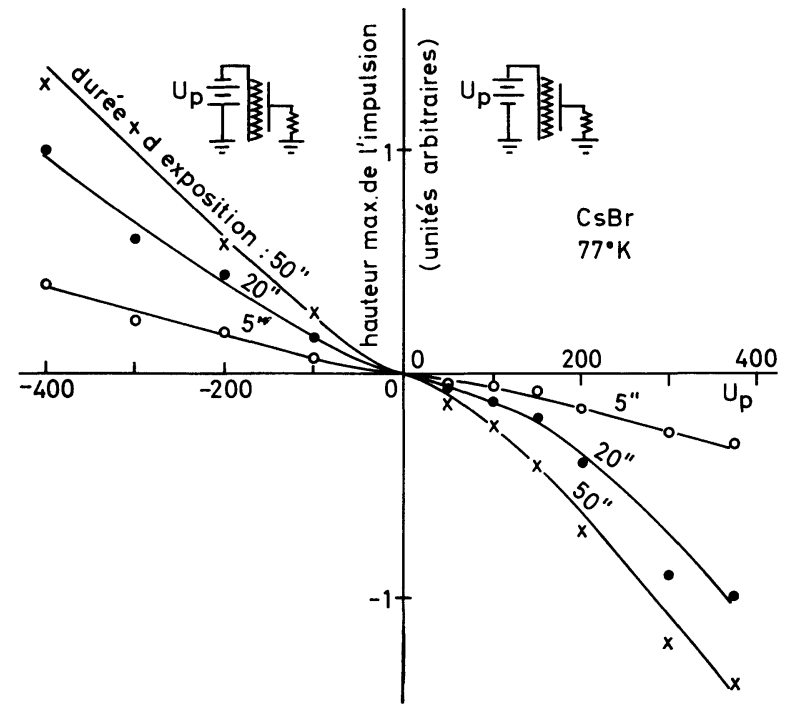

FIG. 4.

$\left({ }^{3}\right)$ Dans notre formule (6), le coefficient 2 a disparu puisque nous ne déduisons $\mu$ qu'à partir d'une mesure $(+B)$.
Quelques instants avant l'apparition de l'étincelle filtrée au préalable pour ne laisser passer que les radiations situées dans la queue de la bande d'absorption, la tension $U_{p}$ est supprimée et la couche résistive est mise à la masse. Nous avons alors observé le courant de photodiffusion en fonction de la tension $U_{p}$ pour des durées variables d'exposition à la lumière rouge. Les électrons, libérés durant le temps d'exposition à cette lumière, sont attirés vers l'électrode positive qui est, suivant le sens d'application de $U_{p}$, soit la couche résistive, soit l'électrode métallique. Lors de l'apparition de l'éclair, la libération d'électrons ne se fera donc plus de façon rigoureusement exponentielle, mais un grand nombre sera libéré près de l'électrode positive. Les électrons libérés par le flash se meuvent alors sous l'influence de la diffusion et du champ électrique créés par les charges piégées. C'est ce mouvement que nous détectons.

Nous tirons de la figure 4 les conclusions suivantes :

$1^{0}$ Pour des durées croissantes d'exposition à la lumière rouge, l'effet des charges spatiales s'accroît et ce, au moins jusqu'à des durées de l'ordre de la minute.

$2^{\circ}$ La réponse de photodiffusion n'est pas une fonction linéaire de la tension $U_{p}$; la pente des courbes croît au fur et à mesure que $U_{p}$ augmente. Nous avons pu aussi observer qu'une charge d'espace ainsi créée peut subsister jusqu'à 30 à $40 \mathrm{~s}$, même en présence d'une lumière dépolarisante (en l'absence de champ électrique) qui engendre un courant de photodiffusion concourant à égaliser les charges.

Cette charge d'espace est créée non pas par des électrons provenant de centres $F$, mais bien par des électrons qui avaient été capturés au préalable par des pièges peu profonds et que la lumière dépolarisante a remis dans la bande de conduction. En effet, l'énergie des photons provenant de la lumière dépolarisante est trop faible pour faire passer un électron d'un centre $F$ dans la bande de conduction.

Ces expériences nous ont prouvé :

$1^{\circ} \mathrm{Qu}$ 'il existait des pièges peu profonds capables de capturer des électrons et de créer une charge d'espace relativement stable.

$2^{\circ}$ Qu'il est possible, à l'aide d'une lumière rouge, de dépolariser le cristal, les temps d'exposition nécessaires pour supprimer toute charge d'espace pouvant aller jusqu'à la minute.

G'est pour cette dernière raison que dans nos expériences sur la mobilité, avant l'application d'une étincelle, le cristal était illuminé par une lumière rouge. Au moment de l'apparition de l'étincelle, nous étions en droit de supposer qu'aucune charge spatiale n'existait dans le cristal et que cette situation persistait aux premiers instants du signal.

IV. Résultats expérimentaux. - Notre dispositif est essentiellement semblable à celui décrit dans [1].

Une résistance en carbone de $40 \Omega$, installée dans 
la cellule contenant le cristal et le plus près possible du cristal, nous permet de mesurer la température entre 10 et 50 K. Son étalonnage s'est fait par comparaison avec une résistance étalon en platine. On peut estimer la précision de mesure sur la température à environ $\pm 1^{\circ}$. La capacité totale d'entrée du préamplificateur, que nous avons réduite au maximum, est de $21,8 \mathrm{pF}$ et la sensibilité de mesure est de $Q=5,5 \times 10^{-15} \mathrm{Cb}$.

En vue d'éprouver notre appareillage, nous avons initialement effectué une série de mesures d'effet Hall dans l'AgCl. Ces résultats ont été comparés avec les résultats publiés en [1]. C'est ce que montre la figure 5. On constate un accord satisfaisant, si l'on excepte un de nos résultats $\left(T=60^{\circ} \mathrm{K}\right.$, $\left.\mu_{H}=400 \mathrm{~cm}^{2} / \mathrm{V} . \mathrm{s}\right)$.

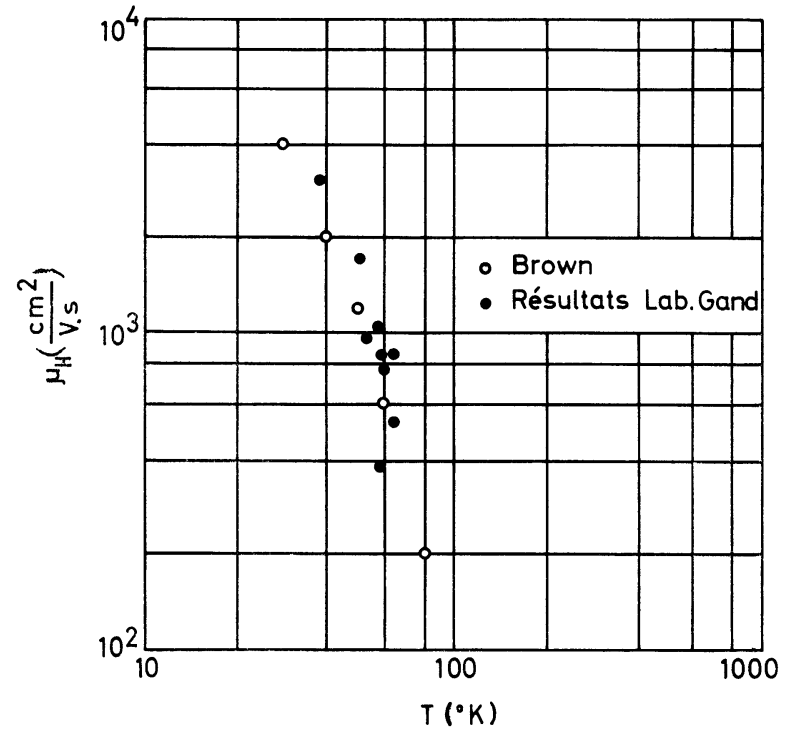

F'IG. 5. - Mobilité de Hall dans AgCl.

Les cristaux de CsBr utilisés provenaient de la firme Korth. Ils ont été colorés additivement suivant la méthode de Van Doorn [8]. Avant chaque expérience, le cristal était chauffé jusqu'à $500^{\circ} \mathrm{C}$, puis refroidi très rapidement. Entre ce dernier moment et l'instant du début de l'expérience, le cristal n'était plus exposé qu'à une lumière rouge se situant hors de la bande $F$. Le spectre d'absorption est semblable à celui donné dans [9]. La concentration en centres $F$ des cristaux utilisés dans chacune des expériences a été estimée à l'aide de la formule de Smakula [10].

Les expériences ont été conduites comme suit :

$1^{0}$ La lumière de l'éclair, non préalablement filtrée (coefficient d'absorption dans $\mathrm{CsBr}$ inférieur à $5 \mathrm{~cm}^{-1}$ ), tombe toutes les $20 \mathrm{~s}$ sur le cristal.

$2^{\circ}$ Durant toute l'expérience, une lumière dépolarisante de $0,675 \mu$ tombe sur le cristal.

$3^{\circ}$ Les valeurs du champ électrique transversal sont de l'ordre d'une centaine de volts par centimètre.

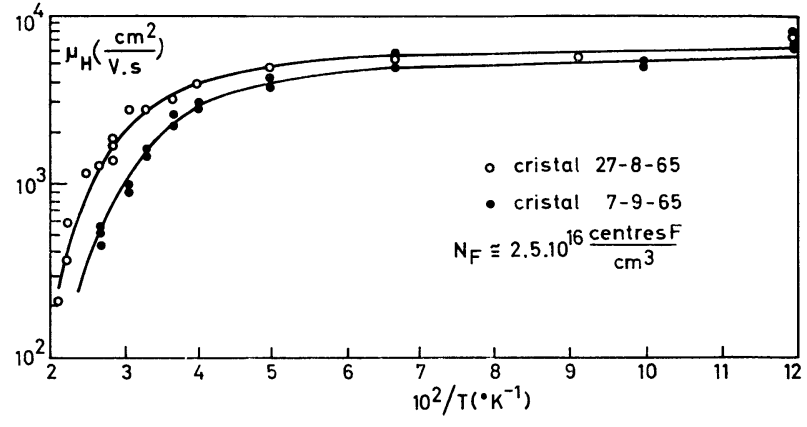

FIG. 6. - Mobilité de Hall dans CsBr.

Nous donnons, figure 6, l'ensemble des résultats obtenus pour deux cristaux. Les mobilités sont calculées par application de (6).

La correction sur la valeur de la mobilité, proposée par Redfield [2] et tenant compte des dimensions finies du cristal, est négligeable vis-à-vis des erreurs expérimentales.

Un cristal de $\mathrm{CsBr}$, de même provenance, a été coloré par irradiation de rayons $\gamma$ à la température de l'azote liquide au C.E.N. à Mol. La sensibilité insuffisante de notre appareil ne nous a pas permis de faire de mesures de mobilité, bien que l'on ait observé des signaux de photoconduction. Nous pouvons estimer à $200 \mathrm{~cm}^{2} / \mathrm{V}$.s la plus petite mobilité mesurable par notre dispositif.

V. Discussion. - D'après le signe du signal de Hall observé dans le $\mathrm{CsBr}$, nous pouvons dire que le courant est produit par des électrons.

A des températures suffisamment élevées mais inférieures à la température de Debye, la mobilité microscopique $\mu_{0}$ est déterminée par l'interaction avec les phonons optiques de grande longueur d'onde, et dans ce cas :

$$
\mu_{H}=\mu_{0}=\mu\left(\mathrm{e}^{\theta / T}-1\right)
$$

où $\mu$ est un coefficient dont l'expression dépend de la théorie prise en considération (Sondheimer, Feynman, Lowe et Pines) et $\theta$ la température de Debye.

Aux températures suffisamment basses, la variation de mobilité microscopique $\mu_{A}$ est donnée par :

$$
\mu_{A}=\frac{8}{3 \pi} \mu_{H}=B T^{-3 / 2}
$$

due à l'interaction avec les phonons acoustiques.

A des températures plus basses encore, la diffusion par les impuretés sera la principale cause de limitation dans les valeurs de la mobilité $\mu_{i}$. Suivant le mécanisme, cette mobilité est calculable théoriquement par des formules telles que celle de Conwell-Weisskopf (diffusion par des particules chargées) ou celle de Appel et Teutsch [18] (diffusion par des dipôles).

Aux températures intermédiaires, la mobilité microscopique est donnée à partir de :

$$
\frac{1}{\mu_{m}}=\frac{1}{\mu_{0}}+\frac{1}{\mu_{A}}+\frac{1}{\mu_{i}} \text {. }
$$


Comme nous n'avons pu trouver dans la littérature la valeur de mobilités pour des températures supérieures et comme nous ne pouvons mesurer des mobilités inférieures à $200 \mathrm{~cm}^{2} / \mathrm{V} . \mathrm{s}$, nous ne pouvons qu'extrapoler très approximativement la partie linéaire de la courbe comprise entre $10^{2} / T$ et $2 \times 10^{2} / T$. Cette extrapolation indique que la valeur de la température de Debye est comprise entre 300 et $420^{\circ} \mathrm{K}$. Cette valeur est en désaccord marqué avec les valeurs déjà publiées pour le $\mathrm{CsBr}$ (tableau I).

\section{TABLEAU I}

Température de Debye

\begin{tabular}{clc}
$\theta\left({ }^{\circ} \mathrm{K}\right)$ & \multicolumn{2}{c}{ RÉFÉRENCES } \\
- & & \\
109 & Joshi et Mitra & {$[11]$} \\
166 & & {$[12]$} \\
73 & Blackman & {$[13]$} \\
136 & Joshi et Mitra & {$[14]$} \\
148,8 & Vallin et coll. & {$[15]$} \\
125 & Dheer et Sharan & {$[16]$} \\
176 & Brown & {$[17]$}
\end{tabular}

Celles-ci ont été obtenues soit à partir de la connaissance expérimentale des constantes élastiques, soit d'après des données spectrales sur l'absorption dans l'infrarouge. Il en résulte une valeur de $\mu$ plus faible que les valeurs habituelles. Cependant, la valeur de $B$ pour $\theta=300$ oK est environ de $5 \times 10^{5} \mathrm{~cm}^{2} / \mathrm{V} . \mathrm{s}$, valeur raisonnable. Gette valeur de $B$ a été déduite par application de la formule (8) dans le domaine de température où l'on peut, en toute première approximation, supposer que seuls les phonons acoustiques limitent la mobilité. Par itération, l'on pourrait déterminer de meilleures valeurs de $\theta, \mu_{i}$ et $B$. Cependant, la précision des mesures rend ce calcul illusoire.

$\mathrm{Au}$ stade actuel des expériences, il nous est impossible de discuter valablement des mécanismes de diffusion qui limitent $\mu_{i}$. Pour ce faire, nous devrons disposer de résultats pour diverses valeurs de concentration du centre $F$ et connaître la nature et la concentration en impuretés dans les cristaux de GsBr.

Remerciements. - Nous tenons à remercier l'I.R.S.I.A. (4) qui nous a permis de réaliser ce travail, les Professeurs Dekeyser et F. Brown pour leurs suggestions et discussions. Le concours qui nous a été apporté par le Laboratoire de Recherches de la M.B.L.E. en la personne de M. Dufour pour les calculs numériques, ainsi que par $\mathbf{M}$. de Baets du C.E.N. Mol pour l'irradiation, a été grandement apprécié.

(4) I.R.S.I.A. : Institut pour 1'Encouragement de la Recherche Scientifique Appliquée à l'Industrie et à l'Agriculture.

\section{BIBLIOGRAPHIE}

[1] Brown (F. C.) et Kobayaschi (K.), Phys. Rev', $1959,113,2$.

[2] Redfield (A. G.), Phys. Rev., 1953, 94, 3.

[3] Brown (F. C.) et Inschauspé (N.), Phys. Rev., 1960, 121, 5 .

[4] Ahrenkiel, (R. K.), Thèse, Illinois, 1963.

[5] Masumi (T.), Ahrenkiei, (R. K.) et Brown (F. C.), Phys. Stat. Sol., 1965, 11, 163.

[6] Burnham (D. C.), Brown (F. C.) et Knox (R. S.), Phys. Rev., 1960, 119, 5.

[7] Fujita (H.), Kobayaschi (K.), KaWai (T.) et Shiga (K.), Phys. Soc. Japan, 1965, 20, 1.

[8] Van DoORN (C. Z.j et Hoven (Y.), Phillips Res. Report, 1956, 11, 479.

[9] Linch (D. W.), Phys. Rev., 1962, 127, 5.
[10] Smakula (Z.), Phys., 1930, 59, 603-614.

[11] Mitra (S. S.) et Joshi (S. K.), Physica, 1960, 26, 284.

[12] Mitra (S. S.) et Joshi (S. K.), Physica, 1960, 26, 825.

[13] Blackman (M.), Hand. Physik, 1955, VII/1, 325.

[14] Joshi (S. K.) et Mitra (S. S.), Proc. Phys. Soc., London, 1960, 76, 295.

[15] Valitin (J.), Beckman (O.) et Salama (K.), J. Applied Physics, 1964, 35, 4.

[16] DheEr (J. D.) et Sharan (B.), Phys. Stat. Sol., 1965, 9, 701 .

[17] Brown (F. C.), Polarons and Excitons, Kuper C. G. et Whitefield, 1963, p. 325.

[18] Appei, (J.) et Teutsch (W. B.), J. Phys. Chem. Solids, 1962, 23, 1521. 